Annuaire suisse de politique de développement

27-1 | 2008

Faits et statistiques 2008

\title{
8. Place financière suisse
}

\section{Gérard Perroulaz}

\section{OpenEdition \\ Journals}

Édition électronique

URL : http://journals.openedition.org/aspd/214

ISSN : 1663-9669

\section{Éditeur}

Institut de hautes études internationales et du développement

Édition imprimée

Date de publication : 1 avril 2008

Pagination : 123-138

ISBN : 978-2-940415-01-4

ISSN : 1660-5934

Référence électronique

Gérard Perroulaz « 8. Place financière suisse », Annuaire suisse de politique de développement [En ligne], 27-1 | 2008, mis en ligne le 02 juillet 2009, consulté le 07 septembre 2020. URL : http:// journals.openedition.org/aspd/214 


\section{Place financière suisse*}

E

$N$ GÉRANT environ le tiers des actifs financiers privés mondiaux placés à l'étranger, le secteur financier suisse a une grande importance dans l'économie du pays. Pour cette raison, la Suisse doit aussi veiller à ce que la place financière ne soit pas utilisée pour le blanchiment d'argent provenant d'activités criminelles. Si l'arsenal législatif a été beaucoup amélioré depuis le début des années 1990 pour mieux lutter contre le blanchiment, la criminalité internationale et la corruption, deux principaux dossiers d'actualité mettent en évidence les champs de tension entre politique économique (défense des intérêts de l'économie suisse) et politique de développement (dont la lutte contre l'évasion fiscale et la lutte contre la criminalité internationale). D'une part, les organisations non gouvernementales de développement contestent la distinction particulière en Suisse entre la soustraction fiscale (omission de déclaration de revenus au fisc, non punissable) et l'escroquerie fiscale (falsification de documents pour échapper au fisc, punie sur le plan pénal). A part quelques concessions accordées dans ce domaine envers les Etats-Unis et l'Union européenne, la Confédération, la majorité du Parlement et le secteur bancaire réaffirment l'importance du maintien de cette distinction pour la compétitivité de la place financière suisse. D'autre part, dans le domaine de la restitution d'argent détourné par d'anciens dictateurs ou autres dirigeants de pays en développement, plusieurs cas de procédures en cours ont mis en évidence les difficultés d'application de l'entraide judiciaire (lenteurs de la procédure).

\subsection{Place financière suisse, fuites fiscales et accords bilatéraux}

\subsubsection{Importance économique de la place financière suisse}

Le secteur bancaire représente environ $9 \%$ du produit intérieur brut (PIB) total de la Suisse ${ }^{1}$. Le montant total stocké par la clientèle dans les banques suisses s'élèverait à 5374 milliards de francs en 2007, dont 3183 milliards de dépôts de titulaires étrangers, la clientèle internationale détenant ainsi près de $60 \%$ de la fortune totale gérée en Suisse ${ }^{2}$. Ce montant total de dépôts de la clientèle des banques est en nette augmentation ces dernières années, puisque le montant total des titres stockés est passé de 3532 milliards de francs en 2004 à 5374 milliards en 2007. Selon l'Association suisse des banquiers (ASB), le secteur financier suisse gérerait plus d'un tiers de tous les fonds mondiaux placés offshore, ce qui montre l'importance de la place financière suisse dans la gestion de fortune privée au niveau mondial ${ }^{3}$. La

* Par Gérard Perroulaz, chargé de recherche et d'enseignement à l'Institut universitaire d'études du développement.

1 Département fédéral des finances (DFF), Chiffres clés relatifs à la place financière suisse, Berne, DFF, août 2007.

2 Banque nationale suisse, Bulletin mensuel de statistiques économiques, juin 2007. Chiffres: état fin mai 2007.

3 ASB, La Suisse et sa place financière, Bâle, ASB, février 2006. 
Suisse serait le plus grand gestionnaire de fortune du monde. Les banques en Suisse sont davantage spécialisées dans la gestion de fortune qu'ailleurs. Les opérations liées à la gestion de fortune représentent $40 \%$ du revenu des banques suisses, les autres sources de revenus étant l'activité bancaire classique et le négoce des titres. Le canton de Zurich réalise $45 \%$ de la valeur ajoutée des banques, Genève $15 \%$ et le Tessin $7 \%{ }^{4}$. Selon les estimations du Département fédéral des finances, les impôts sur le revenu et sur les entreprises versés par le secteur financier (banques et assurances) s'élèveraient à $10 \%$ au moins du total des recettes fiscales en Suisse ${ }^{5}$. Le secteur emploie $3 \%$ à 3,5\% des emplois totaux en Suisse, avec près de 128000 employés du secteur bancaire en $2006^{6}$.

Les statistiques de la Banque nationale suisse (BNS) concernant les opérations fiduciaires des banques suisses pour le compte de clients étrangers donnent une idée (partielle) de l'argent géré par une partie des banques suisses. En 2006, les engagements totaux à titre fiduciaire de 95 banques établies en Suisse se sont élevés à 328,3 milliards de francs suisses ${ }^{7}$. La grande majorité des placements fiduciaires sont effectués pour des clients d'économies avancées. On constate cependant aussi des opérations fiduciaires importantes pour le compte de clients de pays beaucoup plus pauvres. Les engagements nets s'élèvent par exemple à 6,7 milliards de francs pour des clients du Liberia, 1,5 milliard de francs provenant de l'Uruguay, 882 millions de francs du Pakistan, 780 millions du Kenya, 237 millions de Côte d'Ivoire et 221 millions du Kazakhstan.

[D] Annuaire 2008, $\mathrm{n}^{\circ}$ 1, partie «Statistiques », chapitre B, graphique B.6.

Les fonds importants placés dans les banques suisses entraînent un risque d'utilisation de la place financière pour blanchir de l'argent issu d'activités criminelles. La Confédération a donc un rôle de contrôle dans ce domaine, et le secteur bancaire affirme aussi vouloir éviter des affaires pouvant ternir sa réputation. De nombreux renforcements des lois ont été adoptés depuis le début des années 1990 pour améliorer la lutte contre le blanchiment d'argent, le crime organisé et la corruption et réprimer le financement du terrorisme.

[D] Annuaire 2006, $\mathrm{n}^{\circ}$ 1, tableau sur l'amélioration de l'arsenal législatif en Suisse pour la lutte contre le blanchiment et la criminalité, p. 124.

Le secret bancaire est ainsi levé lors de soupçons d'activités délictueuses et l'entraide judiciaire internationale en matière pénale permet de fournir des informations à des Etats tiers qui soupçonnent que des fonds issus du crime soient dans des banques suisses.

\subsubsection{La Suisse ne punit pas toutes les formes de fuites fiscales}

Pour l'Association suisse des banquiers, «la Suisse part du principe que chaque citoyen (et non sa banque) répond personnellement de son obligation fiscale vis-àvis de l'Etat. Elle se fonde en cela sur le principe de la déclaration spontanée» ${ }^{8}$.

\footnotetext{
Martina Schriber, «Le secteur bancaire suisse : une histoire à succès, mais aussi à risque », La Vie économique (SECO), $\mathrm{n}^{\circ}$ 4, 2007.

Département fédéral des finances, op. cit.

BNS, Les banques suisses. 2006, Zurich, BNS, 2007.

Ibid., tableau 38.

ASB, op. cit., p. 7.
} 
Les banques suisses ne sont ainsi pas tenues d'informer les autorités fiscales du montant des intérêts versés à un client sur son compte d'épargne. La soustraction fiscale ne constitue pas dans le droit suisse une infraction pénale. L'escroquerie fiscale, soit la falsification de documents (livres de comptes, bilans, comptes de résultat, bulletins de salaire) dans le but de frauder le fisc, est en revanche pénalement sanctionnée 9 .

L'entraide judiciaire internationale n'est donc pas accordée par la Suisse pour les fuites fiscales, sauf en cas d'escroquerie fiscale. La simple «omission» de déclarer des revenus ou de la fortune à l'étranger et le placement de cet argent dans une banque suisse ne sont ainsi pas punissables. C'est cette distinction particulière du droit suisse entre soustraction et escroquerie fiscale qui est régulièrement dénoncée par des organisations non gouvernementales (ONG) suisses, dont notamment la Déclaration de Berne. Celles-ci souhaitent que la Suisse prenne une part plus active à la lutte contre les fuites de capitaux en punissant l'évasion fiscale dans tous les cas. Des propositions de parlementaires cherchant à supprimer purement et simplement la clause d'exclusion du délit fiscal ont été régulièrement repoussées par la majorité des Chambres fédérales. La dernière tentative de faire de la soustraction fiscale un délit pénalement condamnable et pouvant de ce fait être l'objet d'une entraide judiciaire ${ }^{10}$ a été rejetée par le Conseil national le 12 mars 2007.

C'est la Loi fédérale sur l'entraide internationale en matière pénale qui prévoit à son article 3 que «la demande [d'entraide] est irrecevable si la procédure vise un acte qui paraît tendre à diminuer des recettes fiscales $\gg^{11}$, mais des traités bilatéraux pourraient prévoir des règles plus larges pour l'entraide car ce n'est pas le secret bancaire en soi qui fait obstacle à des demandes de l'étranger. A la suite de pressions d'autres pays, la Suisse a été obligée, ces dernières années, d'accepter un assouplissement de la loi dans certains cas.

Les Conventions de l'ONU sur la répression du financement du terrorisme, de la criminalité transnationale organisée et sur la corruption ne prévoient pas la possibilité pour les Etats d'exclure de leur coopération le délit fiscal. L'accord sur l'entraide avec les Etats-Unis prévoit l'obligation pour les banques suisses d'identifier les citoyens américains parmi leurs clients pour prélever un impôt à la source au profit du fisc américain ${ }^{12}$; puis, la Suisse a accepté de donner des informations de nature fiscale dans le domaine de la lutte contre la criminalité et la lutte contre le financement du terrorisme avec ce pays. Dans le cadre des accords bilatéraux entre la Suisse et l'Union européenne sur la lutte contre la fraude (accords de Schengen et de Dublin), la Suisse a dû accepter une collaboration dans le domaine de la fiscalité indirecte (TVA notamment), même dans le cas de simple évasion fiscale ${ }^{13}$. Les traités bilatéraux conclus ces dernières années avec des pays en développement ne prévoient par contre aucune exception, notamment dans les accords bilatéraux sur l'entraide judiciaire.

$9 \quad$ Ibid., pp. 7-8.

10 05.3659. Motion. Faire de la soustraction d'impôt un délit, déposée au Conseil national par Heiner Studer le 7 octobre 2005.

11 Loi fédérale sur l'entraide internationale en matière pénale (RS 351.1).

12 Traité entre la Confédération suisse et les Etats-Unis d'Amérique sur l'entraide judicaire en matière pénale (RS 0.351.933.6).

13 Accord de coopération entre la Confédération suisse, d'une part, et la Communauté européenne et ses Etats membres, d'autre part, pour lutter contre la fraude et toute autre activité illégale portant atteinte à leurs intérêts financiers (FF 2004 6127). 


\subsubsection{Traités bilatéraux d'entraide judiciaire en matière pénale}

Le Parlement a adopté en 2007 de nouveaux traités d'entraide judiciaire en matière pénale avec le Mexique ${ }^{14}$ et le Brésil ${ }^{15}$. Concernant l'Amérique latine, des traités bilatéraux semblables sont déjà en vigueur avec le Pérou (1998) et l'Equateur (1999) et en négociation avec l'Argentine ${ }^{16}$. Le Conseil fédéral a approuvé le 28 novembre 2007 le message concernant le traité d'entraide judiciaire avec le Chili ${ }^{17}$. Ces traités permettent de renforcer la lutte contre la criminalité internationale, notamment dans les domaines de la corruption, la criminalité économique, le trafic de stupéfiants et la traite des êtres humains.

Dans son message présenté aux Chambres fédérales concernant le Brésil, le Conseil fédéral relève que la clause qui exclut les demandes d'entraide pour les infractions fiscales «a fait l'objet de négociations ardues, la délégation brésilienne ayant longuement insisté pour obtenir l'entraide en matière fiscale. Le compromis agréé par les deux délégations prévoit que l'entraide judiciaire peut être accordée si la procédure vise une escroquerie en matière fiscale $»^{18}$. Le conseiller national Carlo Sommaruga (PS/GE) a déposé une interpellation pour éclaircir ce point et demander au Conseil fédéral pourquoi les délégations semblent négocier dans les traités bilatéraux des mesures plus drastiques que celles prévues dans la loi sur l'entraide judiciaire. Dans sa réponse, le Conseil fédéral relève que les traité bilatéraux récents prévoient explicitement l'entraide en cas d'escroquerie fiscale, mais qu'il ne souhaite pas étendre à d'autres pays les exceptions prévues avec les Etats-Unis ou avec l'Union européenne ${ }^{19}$.

\subsubsection{Coopération internationale en matière de police}

La coopération internationale pour mieux lutter contre la criminalité transfrontalière repose sur trois types d'actions:

- la coopération multilatérale par le biais d'Interpol, avec l'échange d'informations policières et les avis de recherche internationaux;

- la coopération multilatérale avec les Etats européens, selon l'accord de Schengen ${ }^{20}$ et par le biais d'Europol, entré en vigueur le $1^{\text {er }}$ mars 2006 (lutte contre les organisations criminelles);

14 Conseil fédéral, Message concernant le Traité d'entraide judiciaire entre la Suisse et le Mexique du 25 octobre 2006 (FF 20068651 ).

15 Conseil fédéral, Message concernant le Traité d'entraide judiciaire entre la Suisse et la République fédérative du Brésil du 28 février 2007 (FF 2007 1903), adopté au Parlement lors de la session d'hiver 2007.

16 Des traités bilatéraux d'entraide judiciaire existent aussi avec les Etats-Unis, le Canada, les Philippines, la Thaillande, l'Australie, Hongkong et l'Egypte.

17 Département fédéral de justice et police, Lutte contre la criminalité : renforcement de la collaboration entre la Suisse et le Chili. Le Conseil fédéral approuve le message concernant le traité d'entraide judiciaire conclu par les deux Etats, communiqué de presse, 28 novembre 2007.

18 Conseil fédéral, Message concernant le Traité d'entraide judiciaire entre la Suisse et la République fédérative du Brésil, op. cit., p. 1911.

19 07.3353. Interpellation. Fraude fiscale. Les négociateurs suisses d'accords bilatéraux d'entraide pénale au-dessus de la loi ?, déposée au Conseil national par Carlo Sommaruga le 19 juin 2007, comprenant la «Réponse du Conseil fédéral du 29.08.2007».

20 Conseil fédéral, Message relatif à l'approbation des accords bilatéraux entre la Suisse et l'Union européenne, y compris les actes législatifs relatifs à la transposition des accords ("accords bilatéraux II ) du $1^{\text {er }}$ octobre 2004 (FF 20045593 ). 
- les accords bilatéraux de coopération policière.

La Suisse a conclu des accords bilatéraux avec ses pays voisins ainsi qu'avec une série de pays européens. Des accords bilatéraux de coopération policière en matière de lutte contre la criminalité sont en vigueur avec la Hongrie, la Lettonie, la Slovénie et la République tchèque. Ceux avec l'Albanie ${ }^{21}$, la Macédoine ${ }^{22}$ et la Roumanie ${ }^{23}$ ont été approuvés par le Parlement pendant la session de printemps 2007, mais ne sont pas encore entrés en vigueur. Un nouvel accord entre la Confédération suisse et la Bosnie-Herzégovine a été signé le 24 avril $2007^{24}$.

Ces accords ont pour but de renforcer la coopération avec les pays de l'Europe de l'Est et du Sud-Est dans la lutte contre la criminalité. Le Conseil fédéral estime en effet que les menaces contre la sécurité intérieure sont étroitement liées au contexte international et que la lutte contre la criminalité doit être menée en étroite collaboration avec d'autres pays. Or les réseaux criminels d'Europe du Sud-Est, par exemple ceux liés au trafic des stupéfiants, à la traite d'êtres humains et au trafic de migrants, touchent également la Suisse. Ces accords permettent en cas de nécessité la formation de groupes de travail communs, la coordination des interventions opérationnelles, le détachement de durée limitée d'attachés de police sur le territoire de l'autre partie. Ils favorisent la formation et le perfectionnement, et créent des bases légales pour les échanges d'informations. Sont exclus de la coopération les délits politiques, militaires et fiscaux.

\subsection{Lutte contre le blanchiment}

\subsubsection{Modification de la Loi sur le blanchiment d'argent (LBA)}

La Suisse a adopté en 1990 une norme pénale pour lutter contre le blanchiment d'argent (art. 305bis et 305ter du Code pénal) ${ }^{25}$. La Loi sur le blanchiment d'argent de 1998 a permis d'introduire des obligations de diligence pour les intermédiaires financiers. Ceux-ci sont tenus de faire part de leurs soupçons en cas de transactions douteuses.

Le Groupe d'action financière sur le blanchiment de capitaux (GAFI), qui dépend de l'Organisation de coopération et de développement économiques (OCDE), est l'organisme de concertation le plus important en matière de lutte internationale contre le blanchiment de capitaux; la Suisse en est membre

${ }^{21}$ Accord entre le Conseil fédéral suisse et le Conseil des ministres de la République d'Albanie sur la coopération policière en matière de lutte contre la criminalité (FF 2006 2145).

22 Accord entre la Confédération suisse et la République de Macédoine sur la coopération policière en matière de lutte contre la criminalité (FF 2006 2157).

23 Accord entre la Confédération suisse et la Roumanie concernant la coopération pour la lutte contre le terrorisme, la criminalité organisée, le trafic illicite de stupéfiants, substances psychotropes et précurseurs, et autres infractions transnationales (FF 2006 2185).

24 Conseil fédéral, Message concernant l'Accord avec la Bosnie-Herzégovine sur la coopération policière en matière de lutte contre la criminalité du 28 septembre 2007 (FF 2007 7107).

25 La répression pénale du blanchiment d'argent s'appuie aussi sur d'autres dispositions du Code pénal suisse (CP): art. 260ter punissant l'appartenance à une organisation criminelle, art. 260quinquies punissant le financement du terrorisme, art. 69 à 72 réglant la confiscation des produits du crime et art. 102 et 102a sur la responsabilité pénale de l'entreprise. 
depuis 1989. Les 49 recommandations établies par le GAFI constituent des standards internationalement reconnus ${ }^{26}$. Le GAFI surveille les progrès réalisés par les pays membres dans la mise en œuvre de législations conformes à ses 49 recommandations et un système d'évaluations mutuelles permet d'examiner les progrès et lacunes de ces législations ${ }^{27}$.

En octobre 2005, le système suisse de lutte antiblanchiment a fait l'objet d'une évaluation du GAFI pour vérifier dans quelle mesure la législation suisse était conforme aux recommandations révisées de l'OCDE. Dans son rapport, l'OCDE relevait que la Suisse disposait d'un système efficace de lutte contre le blanchiment et que son dispositif répondait largement aux normes internationales. Le rapport mentionnait toutefois certaines lacunes dans les lois suisses.

Le Conseil fédéral a mis en consultation un premier projet de révision de la LBA en janvier 2005, mais il a retiré le projet pour un nouvel examen devant la forte opposition des milieux économiques et bancaires. Ceux-ci considéraient que les mesures proposées par le Conseil fédéral allaient trop loin dans certains domaines.

Le 15 juin 2007, le Conseil fédéral a adopté un nouveau message pour modifier la Loi sur le blanchiment (LBA) et ainsi mettre en œuvre une partie des recommandations du $\mathrm{GAFI}^{28}$. Le projet de loi, qui doit encore avoir l'aval du Parlement, prévoit une douzaine de mesures pour améliorer la lutte contre le blanchiment. Le Conseil fédéral ne souhaite par contre pas «étendre excessivement le cadre réglementaire ${ }^{29}$, de façon à ne pas trop alourdir la charge administrative pour les intermédiaires financiers. Il a donc renoncé à certaines dispositions. A la suite de l'opposition des milieux concernés, le gouvernement n'étend pas la surveillance à de nouveaux secteurs d'activités comme l'immobilier, la vente de bijoux et d'œuvres d'art ou le délit d'initié.

Dans son message, qui reprend une partie des recommandations du GAFI, le Conseil fédéral étend le champ de la LBA à la lutte contre le terrorisme. Il prévoit d'inclure dans la loi de nouvelles infractions préalables au blanchiment: contrebande organisée, falsification de marchandises et piratage de produits. L'intermédiaire financier devra dorénavant obligatoirement communiquer ses soupçons au Bureau de communication en matière de blanchiment d'argent (MROS) dès les premiers contacts avec un nouveau client potentiel, même si la relation d'affaires ne s'établit pas ${ }^{30}$. Autre point nouveau, l'obligation de diligence ne concernera plus les intermédiaires financiers pour de petits montants (clause bagatelle) lors de relations suivies et si la légalité de la relation d'affaires est manifeste. La protection juridique de l'intermédiaire financier a été

26 Le système mis en place sur le plan international et au sein des législations nationales était au début axé essentiellement contre le blanchiment d'argent issu du trafic de la drogue. Le dispositif s'est progressivement étendu à d'autres activités criminelles (organisations criminelles, traite des êtres humains et, depuis 2001, financement des activités terroristes).

27 Les rapports d'évaluation sont disponibles sur le site Internet du GAFI, <http://www.fatf-gafi.org>. La Suisse a été examinée en automne 2005.

28 Conseil fédéral, Message sur la mise en æuvre des recommandations révisées du Groupe d'action financière (GAFI) du 15 juin 2007 (FF 2007 5919).

29 Ibid., p. 5926.

30 Jusqu'à maintenant, l'intermédiaire financier n'était pas tenu de communiquer ses soupçons s'il n'ouvrait pas un compte pour un nouveau client qu'il jugeait douteux, celui-ci pouvant alors s'adresser à une autre banque, sans être sur une liste de suspects. 
renforcée pour éviter des risques de représailles à son encontre lors de dénonciations de cas douteux. Les autorités douanières devront participer à la lutte contre le blanchiment en introduisant un système d'information lors de transports transfrontaliers d'argent liquide. Il n'y aura cependant pas d'obligation de déclarer l'entrée d'argent liquide, mais des contrôles ponctuels seront possibles. L'intermédiaire financier devra identifier les représentants de personnes morales et identifier le but et la nature de la relation d'affaires souhaitée par son client.

[D] Annuaire 2006, $\mathrm{n}^{\circ}$ 1, et Annuaire 2007, $\mathrm{n}^{\circ}$ 1, «Activités du Bureau de communication en matière de blanchiment d'argent (MROS)», respectivement pp. 127-130 et 120-122.

\subsubsection{Nouvelle Loi sur la surveillance des marchés financiers (LFINMA)}

Adoptée par le Parlement en juin 2007, la nouvelle Loi sur la surveillance des marchés financiers ${ }^{31}$ permettra de créer une unique Autorité de surveillance des marchés financiers. Sorte de «superloi» chapeautant d'autres lois, la LFINMA a pour objectif essentiel de regrouper les organes fédéraux de surveillance des banques, des entreprises d'assurance et des autres intermédiaires financiers au sein d'une seule autorité de surveillance. Elle permettra de fusionner la Commission fédérale des banques, l'Office fédéral des assurances privées et l'Autorité de contrôle en matière de lutte contre le blanchiment sous la désignation d' «Autorité fédérale de surveillance des marchés financiers (FINMA)». La nouvelle loi règle non seulement les questions d'organisation, mais énonce aussi des principes de réglementation des marchés financiers, fixe les règles en matière de responsabilité, harmonise les instruments de surveillance et prévoit des sanctions. La FINMA sera un établissement de droit public.

\subsection{Lutte contre la corruption}

Les premiers pas importants dans la lutte contre la corruption transfrontalière ont été effectués en Suisse dans le contexte de l'adoption en 1997 de la Convention de l'OCDE sur la lutte contre la corruption. La Suisse a adopté en décembre 1999 la Loi fédérale sur l'interdiction de déduire fiscalement les commissions occultes, entrée en vigueur au $1^{\text {er }}$ janvier $2001^{32}$. L'action de la Suisse dans le domaine de la lutte contre la corruption porte sur les domaines suivants:

๖ L'adhésion à des accords internationaux de lutte contre la corruption. La Suisse a ratifié, le 31 mai 2000, la Convention de l'OCDE sur la lutte contre la corruption d'agents publics étrangers dans les transactions commerciales internationales. Le 31 mars 2006, elle a ratifié la Convention pénale du Conseil de l'Europe sur la corruption. Les nouvelles dispositions pénales sont entrées en vigueur depuis le $1^{\text {er }}$ juillet 2006. Cette convention va plus loin que celle de l'OCDE puisqu'elle permet de réprimer la corruption passive d'agents publics étrangers ainsi que la corruption dans le secteur privé. La Suisse n'a par contre pas encore signé la Convention civile du Conseil de l'Europe sur la corruption.

31 Conseil fédéral, Message concernant la Loi fédérale sur l'Autorité fédérale de surveillance des marchés financiers (LAUFIN) du $1^{\text {er }}$ février 2006 (FF 2006 2741).

32 Loi fédérale sur l'interdiction de déduire fiscalement les commissions occultes (RO 20002147 ). 
- Le dialogue sur le plan international, notamment par les contributions de la Suisse à la Banque mondiale et aux banques régionales de développement. La Suisse est aussi membre du Global Corporate Governance Forum, fondé par le Groupe de la Banque mondiale et par l'OCDE.

口 Le soutien d'initiatives visant à sensibiliser les acteurs concernés en Suisse aux problèmes de la corruption, notamment les entreprises et l'administration publique, ainsi que l'adoption de clauses anticorruption (dans les accords de coopération et les octrois de l'Assurance suisse contre les risques à l'exportation).

$\checkmark$ Le soutien de projets spécifiques de coopération dans certains pays, pour appuyer la mise en place d'une autorité nationale de lutte contre la corruption, par exemple en Equateur ou à Madagascar, en finançant des campagnes de sensibilisation de la population (au Sénégal, au Bénin et au Niger par exemple) et en soutenant des ONG actives dans la prévention de la corruption (Transparency International, mais aussi des ONG au Burkina et Mozambique).

\subsubsection{Suivi de l'application de la Convention de I'OCDE sur la lutte contre la corruption}

L'OCDE suit régulièrement les progrès réalisés par chaque Etat dans l'application de la convention ${ }^{33}$. En 2004, le Groupe de travail de l'OCDE sur la corruption a examiné le dispositif de lutte contre la corruption de la Suisse et formulé une dizaine de recommandations pour améliorer cette lutte. En mars 2007, la Suisse a présenté son rapport de suivi sur la mise en œuvre des recommandations, que le groupe de travail de l'OCDE a examiné34. Le groupe a constaté que plusieurs de ses recommandations (huit sur dix) n'étaient pas encore pleinement appliquées en Suisse.

Des efforts doivent encore être entrepris, selon l'OCDE, pour assurer une meilleure transparence des comptes des entreprises, l'indépendance des organes de révision comptable et la protection efficace des personnes collaborant avec la justice (notamment la protection des salariés dénonciateurs contre les représailles et le licenciement). Des modifications de lois sont en cours dans plusieurs domaines ${ }^{35}$. Dans son rapport sur le suivi de la mise en œuvre des recommandations, la Suisse relève que pour l'instant jamais encore une entreprise, en tant que personne morale, n'a été condamnée pour faits de corruption (art. 100quater CP). En 2005 et 2006, 23 procédures ont été engagées

33 L'OCDE a publié notamment un premier rapport annuel général sur l'application de la convention: OECD Working Group on Bribery, Annual Report 2006, Paris, OECD, 2007, 26 p.

34 OCDE, Rapport de suivi sur la mise en cuvre des recommandations au titre de la phase 2. Suisse: phase 2. Application de la Convention et de la Recommandation révisée de 1997 sur la lutte contre la corruption d'agents publics étrangers dans les transactions commerciales internationales, Paris, OCDE, 2 mai 2007, 25 p.

35 Dans le domaine de la protection des dénonciateurs (whistleblowing), le Parlement a adopté en mars 2006 (Conseil des Etats) et en juin 2007 (Conseil national) la motion Gysin (03.3212), qui demande au Conseil fédéral de présenter au Parlement un projet de loi garantissant une protection efficace des «donneurs d'alerte» et d'examiner la question de l'obligation pour le personnel fédéral de communiquer à l'autorité compétente des soupçons d'actes de corruption. 
pour faits de corruption d'agents publics étrangers, dont 17 en relation avec le programme des Nations unies «Pétrole contre nourriture». Aucun jugement n'a été encore rendu.

[D] Annuaire 2006, $\mathrm{n}^{\circ}$ 1, «Examen par l'OCDE des pratiques suisses de lutte contre la corruption», pp. 132-133.

\subsubsection{Convention des Nations unies contre la corruption}

Le Conseil fédéral a approuvé le 21 septembre 2007 le message demandant aux parlementaires d'accepter la ratification par la Suisse de la Convention des Nations unies contre la corruption ${ }^{36}$, que la Suisse avait signée le 10 décembre 2003. Cent quarante Etats ont signé cette convention et 90 Etats l'ont ratifiée (état en septembre 2007). Elle est entrée en vigueur le 14 décembre 2005.

Principale nouveauté, la convention de l'ONU est le premier instrument international demandant de manière contraignante la restitution des avoirs acquis illicitement. Pour le reste, elle ne va pas plus loin que les instruments régionaux en la matière (OCDE et Conseil de l'Europe); son adoption par la Suisse ne nécessite donc pas de grandes adaptations du droit suisse, déjà largement compatible avec les nouvelles dispositions.

La nouvelle convention de l'ONU traite d'abord des politiques et pratiques à suivre pour la prévention de la corruption. Elle oblige les Etats parties à réprimer les diverses formes de corruption: ils doivent adopter des normes pénales réprimant le détournement de fonds, l'abus de fonctions, le blanchiment d'argent ou le faux témoignage. La responsabilité pénale des personnes morales est engagée et la protection des témoins doit être assurée.

La convention contient des dispositions sur la coopération internationale et règle la restitution d'avoirs provenant des infractions. Elle introduit l'obligation de restituer ces valeurs quand l'Etat concerné peut fournir la preuve qu'il en était propriétaire avant l'infraction et si les règles traditionnelles de l'entraide internationale sont respectées. Pour la Suisse, cette obligation de restituer les produits du crime est compatible avec la Loi fédérale du 19 mars 2004 sur le partage des valeurs patrimoniales confisquées ${ }^{37}$. La convention prévoit qu'un Etat peut refuser d'octroyer l'entraide judiciaire à des Etats qui ne seraient pas des Etats de droit. Lorsque l'Etat requérant n'est pas à même d'aller au bout de la procédure judiciaire, la convention prévoit une possibilité de conclusion d'un accord bilatéral, et l'Etat requis peut dans ce cas avoir son mot à dire sur l'affectation des fonds, dans la mesure du possible au bénéfice de la population et des victimes de la corruption.

Enfin, la convention de l'ONU définit les mécanismes de mise en œuvre des règles, avec principalement des conférences régulières réunissant les Etats parties à la convention. Les mécanismes de suivi sont nettement moins ambitieux que ceux prévus dans les conventions de l'OCDE ou du Conseil de l'Europe. Dans beaucoup de domaines, notamment en cas de corruption dans le secteur privé, la convention de l'ONU contient des dispositions qui ne sont pas obligatoires, car

36 Conseil fédéral, Message concernant la Convention des Nations unies contre la corruption du 21 septembre 2007 (FF 2007 6931).

37 Loi fédérale du 19 mars 2004 sur le partage des valeurs patrimoniales confisquées (RS 312.4). 
aucun consensus n'a pu être trouvé lors des négociations. L'article 20 de la convention de l'ONU prévoit par exemple le renversement du fardeau de la preuve, l'agent public devant apporter la preuve que l'augmentation substantielle de son patrimoine provient d'une activité licite. Cette disposition va plus loin que la législation suisse, mais elle n'est pas contraignante.

\subsubsection{Rôle des acteurs privés dans la lutte contre la corruption}

Un rapport du Centre d'études pratiques de la négociation internationale (CASIN) analyse le rôle des ONG dans les efforts globaux de lutte contre la corruption $^{38}$. Diverses initiatives de lutte contre la corruption ont été développées ces dernières années sous l'impulsion d'ONG, que ce soit au niveau national, régional ou mondial. La plus connue des ONG internationales actives dans ce domaine est sans doute Transparency International, dont le siège est à Berlin et qui a des branches nationales dans 90 pays du monde, dont la Suisse ${ }^{39}$. Transparency International publie régulièrement des rapports ${ }^{40}$ ainsi que des indicateurs et des classements de la perception de corruption (Corruption Perceptions Index).

L'ONG britannique Global Witness analyse les liens entre corruption, exploitation de ressources naturelles, violations des droits humains et conflits armés ${ }^{41}$. Selon cette organisation, la corruption joue un rôle important pour maintenir des populations entières dans la pauvreté et financer les conflits internes dans des pays riches en matières premières. Global Witness cherche à développer des initiatives communes entre gouvernements, organisations de la société civile et entreprises privées. L'initiative «Publish What You Pay» (PWYP, «Publiez ce que vous payez») réunit plus de 300 acteurs de la société civile pour inciter les grandes entreprises pétrolières transnationales à publier les paiements aux pays en développement pour l'exploitation du pétrole, alors qu'un secret entoure le plus souvent ces transactions ${ }^{42}$. Une autre initiative, l' «Extractive Industries Transparency Initiative»(EITI, Initiative pour la transparence des industries extractives), lancée par le gouvernement britannique, existe dans les secteurs de l'industrie extractive (mines) et de l'exploitation du gaz $^{43}$.

데 Annuaire 2007, $\mathrm{n}^{\circ}$ 2, Gilles Carbonnier, «Comment conjurer la malédiction des ressources naturelles ?», pp. 83-98.

\subsection{Argent des potentats et restitution des avoirs bloqués en Suisse}

Le Comité catholique contre la faim et pour le développement (CCFD) a publié en mars 2007 un rapport qui fait le point de la situation concernant l'argent des potentats placé dans les places financières du Nord ${ }^{44}$. Le CCFD estime que le

38 CASIN, Global Anti-Corruption Efforts: The Role of Non-Governmental Organisations, Geneva, CASIN, June 2007, 50 p.

$39<$ http://www.transparency.org $>$.

40 Transparency International, Global Corruption Report 2007, Cambridge (UK), Cambridge University Press, 2007.

$41<$ <ttp://www.globalwitness.org $>$.

$42<$ http://www.publishwhatyoupay.org $>$.

$43<$ <ttp://eitransparency.org $>$.

44 CCFD, Biens mal acquis... profitent trop souvent. La fortune des dictateurs et les complaisances occidentales, Paris, CCFD, mars 2007, 126 p. 
montant de l'argent détourné par des chefs d'Etat et des dictateurs atteint entre 100 et 180 milliards de dollars au total. Sur cette somme, 4 milliards de dollars auraient été restitués aux pays concernés et 2,7 milliards de dollars seraient encore bloqués. La Suisse a restitué des fonds aux pays concernés pour un total de 1,3 milliard de dollars environ: 658 millions de dollars des fonds Marcos (Philippines), 594 millions de dollars dans le cadre de l'affaire Abacha (Nigeria), 80,7 millions de dollars détournés par Fujimori (Pérou), 21 millions de dollars dans le cadre du détournement de la dette angolaise vers la Russie (Angola), 6,6 millions de dollars des fonds Lazarenko (Ukraine) et 2,4 millions de dollars des fonds Traoré (Mali).

L'étude du CCFD met en évidence l'écart énorme entre les montants (difficiles à estimer) d'argent public détournés, les fonds effectivement bloqués et l'argent restitué. Les ONG mettent ainsi en cause les lenteurs des procédures et les lacunes dans les lois. Comme le relève l'étude, la Suisse fait partie des rares pays qui ont effectivement restitué des fonds. De nombreuses procédures d'entraide judiciaire sont en $\operatorname{cours}^{45}$.

L'analyse des cas en cours et des procédures qui ont permis des restitutions d'avoirs dans les pays concernés laisse apparaitre deux types de problèmes d'application de la législation suisse pour la restitution des avoirs dans les pays d'origine :

- les lenteurs de la procédure d'entraide judiciaire;

- les difficultés d'application de la restitution des fonds si l'on veut s'assurer que l'argent restitué bénéficie bien aux victimes des détournements de fonds publics.

\subsubsection{Obstacles et lenteurs de la procédure d'entraide judiciaire}

La Suisse coopère avec l'étranger lors de demandes d'entraide judiciaire concernant des fonds déposés en Suisse par des anciens dirigeants ou dictateurs. Les règles de base de l'entraide judiciaire doivent être respectées, notamment le principe de la double incrimination: le délit doit être punissable dans l'Etat requérant et en Suisse. Les procédures pénales à l'encontre des anciens dirigeants doivent être menées en bonne et due forme dans le pays requérant, dans le respect des droits de l'homme et des droits de l'accusé pendant le procès.

Or, l'application de ces règles se heurte à plusieurs problèmes. Les systèmes judiciaires peuvent être faibles dans bien des pays (comme en Haïti par exemple) et l'héritage de certaines dictatures lourd à porter lorsque des membres de familles d'anciens dictateurs ou des proches restent au pouvoir ou qu'un procès contre l'ancien dirigeant risque de provoquer des troubles intérieurs. L'Etat requérant peut ainsi ne pas être en mesure de mener à terme la procédure pénale, pour des raisons politiques et par manque de «bonne gouvernance». Dans un tel contexte, un procès «équitable» au sens du droit suisse est parfois irréalisable.

45 Pour en savoir plus sur certains cas précis de détournements de fonds et de procédures de restitution, voir l'étude du CCFD (op. cit.), mais aussi les sites Internet de la Déclaration de Berne, <http://www. ladb.ch>, et de l'Action place financière suisse, <http://www.aktionfinanzplatz.ch>. 
Il est aussi souvent difficile pour le pays lésé de prouver que l'argent retrouvé sur les comptes en Suisse provient bien de l'argent du crime ou de la corruption et pas d'activités légales. Des solutions pourraient être envisagées pour résoudre ce problème, comme par exemple le renversement de la charge de la preuve. Au lieu que le pays concerné doive prouver que l'ex-président a détourné des biens publics pour les mettre à l'étranger, il s'agirait de demander à l'ex-dirigeant de prouver que son argent est «propre» et ne provient pas du détournement d'argent public. Ce renversement du fardeau de la preuve existe déjà dans d'autres cas, comme l'argent de la criminalité internationale: la personne soupçonnée d'avoir placé de l'argent criminel en Suisse doit prouver elle-même que l'argent placé et saisi en Suisse provenait d'activités tout à fait licites. Ce renversement du fardeau de la preuve a ainsi été utilisé dans le cas des fonds Abacha, le «clan Abacha» étant considéré par la Suisse comme une «organisation criminelle». En outre, comme les pays requérants n'ont souvent que des moyens dérisoires pour mener à bien des enquêtes, une assistance technique (par des ONG ou des coopérations du Nord) pourrait également être envisagée pour aider les pays à mener les procédures nécessaires, par exemple sur le modèle d'Eurojust ${ }^{46}$, qui est un point de coordination entre les différentes autorités européennes pour simplifier et améliorer les procédures.

Puisque la preuve formelle de l'origine illicite des fonds placés en Suisse n'a pas été apportée, la Suisse a par exemple décidé de rendre 2,8 millions de francs bloqués à l'ancien premier ministre malgache Tantely Andrianarivo, alors que le gouvernement malgache avait demandé l'entraide judiciaire et condamné l'ancien ministre pour détournement de deniers publics ${ }^{47}$.

Voici deux autres cas d'actualité qui illustrent le problème des lenteurs de la procédure d'entraide:

\section{$\square$ La difficile restitution des fonds Duvalier (Haïti)}

Depuis 1986, 7,6 millions de francs appartenant à la famille Duvalier sont bloqués en Suisse; des procédures sont en cours aussi dans d'autres pays. En 2007, l'affaire revient sur le devant de l'actualité en Suisse, lorsque le Conseil fédéral annonce début juin qu'il devra vraisemblablement lever le blocage des fonds, qui pourraient dans ce cas être restitués au clan Duvalier. Aucune procédure pénale contre les Duvalier n'est en cours en Haïti ; or, la Suisse ne peut rendre des fonds au pays concerné que si une telle procédure existe. Diverses œuvres d'entraide ont réagi en Suisse; elles ont relevé les lacunes juridiques en Suisse et demandé une prolongation du blocage des fonds. Le 22 août 2007, le Conseil fédéral a annoncé que les 7,6 millions de francs seraient bloqués pour une (ultime) période de douze mois supplémentaires. Cette prolongation a été possible après que le président actuel en Haïti eut signalé à la Suisse la ferme volonté du gouvernement haïtien de lancer des procédures contre l'ex-dictateur. Plusieurs observateurs (presse, ONG et experts) ont souligné les risques pour la réputation de la place financière suisse s'il s'avérait que la Suisse doit finalement restituer des fonds à d'anciens dictateurs ${ }^{48}$.

$46<$ <ttp://eurojust.europa.eu>.

47 Swissinfo, Effluves de blanchiment aux narines des Malgaches, 3 mai 2007.

48 Voir par exemple l'article de Mark Pieth «Potentatengelder als Reputationsrisiko für den Finanzplatz Schweiz. Drohendes Debakel im Fall Duvalier», Neue Zürcher Zeitung, 6. Juni 2007. 


\section{$\square$ République démocratique du Congo}

Des fonds Mobutu sont bloqués en Suisse depuis que la République démocratique du Congo (RDC) a adressé une demande d'entraide judiciaire à la Suisse en 1997. Les autorités judiciaires de la RDC n'ont pas été en mesure de fournir toutes les preuves du détournement illicite des fonds et aucun procès n'a eu lieu dans cet Etat encore fragile. Le Conseil fédéral a décidé en décembre 2003 de bloquer trois ans encore les avoirs de Mobutu, pour un montant d'environ 10,8 millions de francs. Après une ultime prolongation et si rien n'avance, les fonds devront être débloqués en novembre 2008.

\subsubsection{Utilisation appropriée des fonds restitués dans les pays d'origine}

Une fois l'accord trouvé avec le pays requérant pour restituer les fonds, il s'agit encore d'avoir des mesures de contrôle et d'accompagnement pour veiller à ce que l'argent restitué soit bien utilisé pour des projets utiles à la population pauvre et qu'il ne disparaisse pas à nouveau dans les méandres de la corruption. Dans ce domaine, les ONG insistent pour qu'il y ait un contrôle démocratique, qui associe les ONG et la société civile du pays concerné, et une transparence réelle sur l'usage des fonds.

Trois exemples d'actualité illustrent le problème de l'utilisation des fonds restitués:

\section{$\square$ Rapports sur l'utilisation des fonds Abacha}

A la suite d'une procédure d'entraide entamée en 1999, la restitution des fonds Abacha au Nigeria s'est opérée de manière novatrice, sous le monitoring de la Banque mondiale et le regard d'une coalition d'ONG. Le Nigeria avait promis que les fonds restitués par la Suisse serviraient à financer une cinquantaine de projets d'infrastructure, d'éducation, de santé et de développement. Fin décembre 2006, la Banque mondiale a publié un rapport d'évaluation sur l'utilisation des fonds Abacha qui révèle certaines failles dans le processus, notamment des difficultés pour vérifier exactement le financement effectif des projets annoncés par le gouvernement du Nigeria (en raison des lacunes dans la comptabilité publique) et un manque d'informations sur la mise en œuvre de ces projets.

Le rapport indépendant des ONG publié en parallèle a mis au jour plusieurs irrégularités dans l'utilisation des fonds restitués, comme de nombreux projets inachevés ou abandonnés. D'autres projets étaient terminés avant même la restitution des fonds ${ }^{49}$. En outre, beaucoup de projets ont été conçus par le gouvernement central sans tenir compte des besoins réels des populations bénéficiaires. La Coalition des ONG pour une restitution juste et transparente des fonds Abacha a dénoncé ces irrégularités et regrette que la population locale n'ait pas suffisamment profité de l'argent restitué. Ce cas démontre à ses yeux le rôle important que peuvent avoir les ONG dans le monitoring et le contrôle de l'utilisation des fonds ${ }^{50}$. Le processus a en effet été suivi de près par une coalition d'ONG en

49 Nigerian Network on Stolen Assets, Shadow Report on the PEMFAR Monitoring Exercise, Benin City, Nigeria, African Network for Environment and Economic Justice, December 2006, 78 p.

50 Sur le rôle des ONG et le monitoring, lire l'article suivant: Aktion Finanzplatz Schweiz, «AbachaKampagne - Forderungen der Zivilgesellschaft umgesetzt », Finanzplatz Informationen, $\mathrm{n}^{\circ}$ 1, 2007, pp. 2-3. 
Suisse, comprenant notamment la Déclaration de Berne, l'Action place financière, Transparency International Suisse et Pain pour le prochain, et par un réseau d'ONG du Nigeria, le Nigerian Network on Stolen Assets (NNSA).

$\mathbb{E}$ Annuaire 2007, $\mathrm{n}^{\circ}$ 1, encadré «Restitution et utilisation des fonds illicites - les fonds Abacha», p. 129.

\section{$\square$ Kazakhstan}

En mai 2007, le Conseil fédéral a approuvé un accord pour rendre au Kazakhstan 120 millions de dollars bloqués à Genève depuis 1999. Selon cet accord, conclu avec les Etats-Unis - l'argent provenant d'un homme d'affaire américain -, la Banque mondiale et le pays concerné, l'argent restitué devrait être utilisé en faveur de programmes de scolarisation pour des enfants défavorisés dans ce pays. Les ONG regrettent que la société civile ne soit pas associée au choix et au monitoring des projets.

\section{$\square$ Angola}

Entre 1997 et 2000, l'entreprise d'Etat de l'Angola Sonangol a versé 774 millions de dollars sur un compte de l'UBS à Genève, compte appartenant à une structure financière, Abalone, fondée par l'homme d'affaires français PierreJoseph Falcone et un milliardaire russe. Ce compte devait permettre de rembourser la dette angolaise envers la Russie, mais seuls 162 millions de dollars ont été versés au gouvernement russe. Le reste s'est dispersé sur des comptes de sociétés financières offshore et sur les comptes des propriétaires d'Abalone. L'une des deux procédures judiciaires ouvertes à Genève a été classée fin 2004 et l'autre est encore en cours. La justice angolaise n'a visiblement pas non plus appuyé les enquêteurs genevois. En septembre 2002, la Confédération suisse a proposé au gouvernement angolais de restituer l'argent saisi à Genève. En octobre 2005, le procureur de Genève, Daniel Zappelli, a donné son accord pour la restitution d'une somme de 21 millions de francs seulement, mais à la fin de 2007 la Suisse et l'Angola n'ont toujours pas réussi à s'entendre sur la manière de gérer cette somme d'argent (notamment au sujet des projets de déminage souhaités par la Suisse $)^{51}$.

\subsubsection{Nouveaux moyens envisagés pour la restitution des avoirs volés}

La Direction du droit international public du Département fédéral des affaires étrangères (DFAE) étudie les moyens de saisir et de bloquer des fonds en dehors des procédures d'entraide judiciaire demandées par la loi, et également les moyens d'améliorer la restitution des fonds détournés ${ }^{52}$. Reste encore à convaincre la majorité du Parlement s'il faut modifier la loi.

Avant la nouvelle Convention des Nations unies contre la corruption, il n'existait pas d'instrument international réglant totalement la question de la restitution de biens de personnes politiquement exposées (PEP) placés illégalement à l'étranger (voir plus haut section 8.3.2). Les conventions internationales de

51 Jean-Claude Huot, «Angola: "L'affaire suisse” », Vers un développement solidaire, $\mathrm{n}^{\circ}$ 193, septembre 2007, pp. 4-6.

52 Voir notamment le document suivant du DFAE: Directorate of Public International Law, Swiss Measures Related to Illicitly Acquired Assets, Federale Department of Foreign Affairs, 2007. 
même que les lois suisses en vigueur ne concernent que la restitution et l'utilisation de fonds issus du trafic de drogue.

Le DFAE étudie des pistes pour garantir la restitution des fonds ${ }^{53}$, tout en relevant que les procédures de restitution doivent être étudiées au cas par cas car il est impossible d'appliquer une unique procédure à tous les cas. Aucun problème particulier ne se pose pour la restitution des fonds lorsque le gouvernement de l'Etat requérant a été élu de façon démocratique. Si des risques de nouveaux détournements de fonds subsistent, il existe plusieurs moyens de contrôle:

- l'utilisation des fonds restitués pour effacer tout ou partie de la dette extérieure des pays concernés;

口 l'tilisation des fonds restitués pour financer des projets concrets de coopération au développement;

๖ la concertation entre les différents pays concernés s'il y a des procédures de demandes de restitution en cours dans plusieurs pays du Nord;

- la création d'un fonds spécial géré et contrôlé par une organisation internationale, telle que la Banque mondiale, une banque régionale de développement ou le Programme des Nations unies pour le développement (PNUD) par exemple.

Sur le plan multilatéral, la Suisse a salué le lancement d'une nouvelle initiative en faveur de la restitution des avoirs volés: la Banque mondiale et l'Office des Nations unies contre la drogue et le crime (ONUDC) ont annoncé en septembre 2007 une action internationale dans ce domaine ${ }^{54}$. Dans le cadre de cette initiative, les pays en développement recevront un soutien pour améliorer leurs capacités en vue de la restitution des fonds (par exemple dans le domaine de l'entraide judiciaire internationale) et échanger des expériences dans le domaine de la gestion des fonds restitués afin que ceux-ci profitent aux plus pauvres.

La Suisse s'est associée à diverses initiatives pour s'atteler à la question de la restitution de fonds. L' «Initiative de Lausanne» a permis dès 2001 de mettre sur pied une plate-forme de dialogue entre les principales places financières pour harmoniser les procédures de restitution de fonds; les pays en développement sont intégrés dans cette initiative depuis 2006. Les agences de coopération au développement de la Suisse (Direction du développement et de la coopération DDC), de l'Allemagne, du Liechtenstein et du Royaume-Uni cofinancent les activités d'un institut spécialisé dans cette question, l'International Centre for Asset Recovery (ICAR) ${ }^{55}$, créé à 1'Université de Bâle et intégré dans l'Institut sur la gouvernance.

53 Ibid., pp. 8-10.

54 SECO, «Nouvelle initiative de la Banque mondiale pour la restitution des avoirs volés », Newsletter, $\mathrm{n}^{\circ}$ 2, 2007, pp. 3-4.

$55<\mathrm{http}: / /$ www.baselgovernance.org/icar>. 


\section{SOURCES}

Financial Action Task Force (FATF), Annual Report 2006-2007, Paris, OECD, 29 June 2007, 14 p.

Conseil fédéral, Message concernant la Loi fédérale sur l'Autorité fédérale de surveillance des marchés

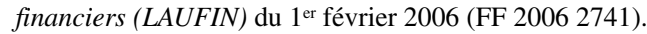

Conseil fédéral, Message sur la mise en cuvre des recommandations révisées du Groupe d'action financière (GAFI) du 15 juin 2007 (FF 2007 5919).

SECO, La Vie économique, $\mathrm{n}^{\circ}$ 11, 2006 (plusieurs articles sur la surveillance des marchés financiers).

Comité catholique contre la faim et pour le développement (CCFD), Biens mal acquis... profitent trop souvent. La fortune des dictateurs et les complaisances occidentales, Paris, CCFD, mars 2007, 126 p.

Déclaration de Berne, Vers un développement solidaire, dossier De la prédation à l'évasion fiscale, $n^{\circ} 193$, septembre 2007.

Plate-forme paradis fiscaux et judiciaires, Paradis fiscaux et judiciaires. Cessons le scandale!, Paris, Secours catholique - Caritas France, avril 2007, 40 p.

\section{SITES INTERNET}

Action place financière suisse: $<\mathrm{http}: / / \mathrm{www}$.aktionfinanzplatz.ch>.

Administration fédérale des finances: <http://www.efv.admin.ch>, et notamment l'Autorité de contrôle en matière de lutte contre le blanchiment d'argent: <http://www.gwg.admin.ch>.

Association suisse des banquiers: $<$ http://www.swissbanking.org $>$.

Commission fédérale des banques: $<$ http://www.cfb.admin.ch $>$.

Déclaration de Berne : <http://www.ladb.ch $>$.

International Centre for Asset Recovery: <http://www.baselgovernance.org/icar>.

OCDE, Groupe d'action financière sur le blanchiment de capitaux : <http://www.fatf-gafi.org>, et lutte contre la corruption: <http://www.oecd.org/bribery $>$.

Office fédéral de la police: <http://www.fedpol.admin.ch>, notamment le Bureau de communication en matière de blanchiment (MROS).

Réseau (d'ONG) pour la justice fiscale: <http://www.taxjustice.net>.

Transparency International Suisse: $<\mathrm{http}: / / \mathrm{www} \cdot$ transparency.ch $>$.

U4 Anti-Corruption Resource Centre: <http://www.u4.no>. 